\section{Infektionen im Krankenhaus: Rationaler Antibiotikaeinsatz bewahrt vor Resistenzen}

\author{
Stationäre Patienten ziehen sich seltener Infektionen mit resistenten Keimen \\ oder Clostridium difficile zu, wenn im Krankenhaus ein Antibiotic-Steward- \\ ship-Programm befolgt wird.
}

$S^{t+m}$ trategien zum rationalen Einsatz von Antibiotika, sogenannte AntibioticStewardship(ABS)-Programme, reduzieren in Krankenhäusern nicht nur Antibiotikaverordnungen und Kosten. Laut einer Metaanalyse von Mitarbeitern der Universitätsklinik Tübingen kommen die Programme auch direkt den Patienten zugute, indem sie die Ausbreitung von resistenten Bakterien und Clostridium (C.) difficile verhindern.

In der Analyse haben die Mediziner um David Baur Daten aus 32 Studien ausgewertet, die mehr als neun Millionen Patiententage abdecken. Bei den Studien, von denen nur zwei eine gute und 26 eine mittlere Qualität hatten, handelte es sich mehrheitlich um Vorher-nachher- und Kohortenstudien. Die häufigsten ABS-Interventionen waren infektiologische Prüfungen (Audits) sowie eine restriktive Verordnungspolitik. Bei circa $30 \%$ der Studien wurden zusätzlich Maßnahmen zur Infektionskontrolle eingesetzt.

Infektionen und Kolonisierungen mit folgenden Erregertypen waren nach der Einführung von ABS-Programmen signifikant reduziert: Multiresistente gramnegative Erreger gingen um $51 \%$, ESBL(Extended-Spectrum BetaLactamase)-bildende um $48 \%$ und Carbapenem-resistente um $43 \%$ zurück. Bei Methicillin-resistentem Stapyhlococcus aureus (MRSA) sank die Inzidenz um $37 \%$. C. difficile wurde um $32 \%$ seltener festgestellt. Lediglich bei Aminoglykosid- und Chinolonresistenz waren die Häufigkeiten nur numerisch, aber nicht statistisch signifikant verringert. Allerdings war laut Autoren zwischen den einzelnen Studien eine „substanzielle Heterogenität“ festzustellen. Die Differenzen konnten zumindest teilweise auf Unterschiede in den Resistenzmustern, der Art der ABS-Intervention und der Studiendauer zurückgeführt werden. Die ABS-Programme waren in hämatervention. ene). kungslos. grammen. hochstehende Studien nötig.

Baur D et al. Effect of antibiotic stewardship on antibiotic-resistant bacteria and Clostridium analysis. Lancet Infect Dis. 2017; online 16. Juni; https://doi.org/10.1016/S1473-3099(17)30325-0

\section{Hier steht eine Anzeige.}

toonkologischen Abteilungen besonders erfolgreich, mit einem Rückgang der Inzidenz resistenter Keime um 59\%. Aber auch Patienten auf Intensivstationen $(-23 \%)$ und in medizinischen Abteilungen $(-22 \%)$ profitierten von der In-

Durch die Kombination von ABS mit Maßnahmen für eine verbesserte Handhygiene konnte die Rate von Besiedlungen/Infektionen mit resistenten Bakterien weiter gesenkt werden (-66\% anstatt $-17 \%$ ohne eine intensivierte Handhygi-

Die Wirkung des ABS hing zu einem großen Teil von den Programminhalten ab. Drei Interventionen waren mit einer signifikanten Reduktion von Resistenzen assoziiert: Antibiotika-Cycling, das heißt der schnelle Wechsel verschiedener Antibiotika (-51\%), infektiologische Audits und Feedback (-34\%) sowie eine restriktive Verordnungspraxis $(-23 \%)$. Die Implementierung von Leitlinien und die Fokussierung auf eine einzige Antibiotikaklasse blieben dagegen wir-

„ABS-Programme haben eine Schlüsselrolle bei der Bekämpfung von Antibiotikaresistenzen, insbesondere von Mehrfachresistenzen bei gramnegativen Keimen", betonen Baur et al. Wegen des synergistischen Effekts empfehlen sie die Implementation in Krankenhäusern in Kombination mit Handhygiene-Pro-

Um den Nutzen unterschiedlicher Programme in Abhängigkeit von der Resistenzlage besser beurteilen zu können, seien jedoch weitere und qualitativ

\section{Dr. Beate Schumacher} the incidence of infection and colonisation with difficile infection: a systematic review and meta- 